\title{
Behaviour change interventions improve maternal and child nutrition in sub-Saharan Africa: a systematic review
}

\author{
D. Watson ${ }^{1}$, P. Mushamiri ${ }^{2}$, P. Beeri ${ }^{3}$, T. Rouamba ${ }^{4}$, S. Jenner ${ }^{5}$, S.H. Kehoe ${ }^{1,5}$, K.A. Ward ${ }^{1,5,6}$, \\ M. Barker ${ }^{5,6,7,8}$, W. Lawrence ${ }^{5,7}$ and the INPreP Study Group \\ ${ }^{1}$ Global Health Research Institute, Human Development and Health, Faculty of Medicine, University of Southampton, \\ Southampton, UK, \\ ${ }^{2}$ SAMRC Centre for Health Economics and Decision Science, PRICELESS, University of the Witwatersrand, School \\ of Public Health, Faculty of Health Sciences, Johannesburg, South Africa, \\ ${ }^{3}$ Navrongo Health Research Centre, Ghana Health Service, Ghana, \\ ${ }^{4}$ Clinical Research Unit of Nanoro, Institute for Research in Health Sciences, National Center for Scientific and \\ Technological Research, Ouagadougou, \\ ${ }^{5}$ MRC Lifecourse Epidemiology Unit, University of Southampton, Southampton, UK, \\ ${ }^{6}$ School of Public Health, Faculty of Health Sciences, University of the Witwatersrand, Johannesburg, South Africa, \\ ${ }^{7}$ NIHR Southampton Biomedical Research Centre, University Hospitals Southampton NHS Foundation Trust, Southampton, UK and \\ ${ }^{8}$ School of Health Sciences, Faculty of Life and Environmental Sciences, University of Southampton, UK
}

Evaluation of the impact of nutrition-specific and nutrition-sensitive interventions on maternal and child nutrition and health in sub-Saharan Africa are inconclusive ${ }^{(1-3)}$. Many of these interventions do not consider the effect of the context in which interventions are carried out and the impact this has on women's ability to implement the behaviour changes often required. Using behavioural components in intervention design may make interventions more effective and outcomes more predictable. In relation to the COM-B model, carefully designed interventions enhance women's Capability, work within her context to offer Opportunities, and support Motivation for changing Behaviour ${ }^{(4)}$. This systematic review aimed to answer the question: Are nutrition-specific and nutrition-sensitive interventions more effective in improving maternal and child nutrition in sub-Saharan Africa if they include behaviour change components?

Six medical, social science and African databases were searched systematically, using MeSH and free text terms, for articles describing nutrition-specific and nutrition-sensitive behaviour change interventions published in English until November 2020. All titles, abstracts and full-text papers were screened twice. Data extraction and quality assessments were based on Centre for Reviews and Dissemination guidelines. Key behaviour change components of the effective interventions were identified and mapped onto the COM-B model and Behaviour Change Wheel. PROSPERO registered (135054).

The search yielded 1127 articles: 64 from 42 interventions met inclusion criteria, ranging from low to high risk of bias. Many that applied behaviour change theory, communication or counselling resulted in significant improvements in maternal and child nutrition status. Outcomes included improvements in infant body composition, household dietary intake and maternal psychosocial measures. Interventions that had more than two behaviour change components, including persuasion, incentivisation, environmental restructuring tended to have the most positive outcomes.

To the best of our knowledge, this is the first systematic review of behaviour change interventions designed to improve maternal and child nutrition in sub-Saharan Africa. The studies included in the review showed that many nutrition-specific and nutrition-sensitive interventions based on behaviour change theory or delivering behaviour change communication or counselling improved mother and child anthropometric indices, diet or psychosocial outcomes. The components of all the interventions included in this review were mapped onto the COM-B model to elucidate mechanisms of action. Specifying the behaviour required in a nutrition intervention and designing the intervention to address participants' Capabilities, Opportunity and Motivations could increase the effectiveness of such interventions.

\section{Acknowledgements}

We would also like to thank the INPreP group for their contributions to this work: Engelbert A. Nonterah, Abraham Oduro, Cornelius Debpuur, James Adoctor, Paul Welaga, Edith Dambayi, Esmond W. Nonterah, Winfred Ofosu, Doreen Ayibisah, Maxwell Dalaba, Samuel Chatio (Navrongo Health Research Centre); Hermann Sorgho, Palwendé R. Boua, Adelaïde Compaoré, Kadija Ouedraogo, Karim Derra, Aminata Welgo, Halidou Tinto (Clinical Research Unit of Nanoro); Karen J. Hofman, Susan Goldstein, Agnes Erzse, Aviva Tugendhaft, Winfreda Mdewa, Ijeoma Edoka (SAMRC Centre for Health Economics and Decision Science, PRICELESS); Mark Hanson, Marie-Louise Newell, Keith M. Godfrey, Caroline Fall, Polly Hardy-Johnson (Faculty of Medicine, University of Southampton); Shane Norris, Emmanuel Cohen, Stephanie Wrottesley (SAMRC Developmental Pathways for Health Research Unit). We would also like to thank Paula Sands at the University of Southampton Health Sciences library for her support in the search strategy.

\section{References}

1. Keats EC, Das JK, Salam RA, et al. (2021) Lancet Child Adolesc Health 5, 367-384.

2. Heidkamp RA, Piwoz E, Gillespie S, et al. (2021) Lancet 397, 1400-1418.

3. Victora CG, Christian P, Vidaletti LP, et al. (2021) Lancet 397, 1388-1399.

4. Michie S, Van Stralen MM \& West R (2011) Implement Sci 6(1), 42. 